\title{
Severe extrapulmonary complications in Mycoplasma pneumoniae infection - case study
}

\author{
Cristina Gabriela Mandric ${ }^{1}$, Otilia-Elena Frasinariu ${ }^{1,2}$, Nistor Nicolai ${ }^{1,2}$, Ingrith Miron ${ }^{1,2}$ \\ 1"Sf. Maria" Children's Emergency Hospital, lasi, Romania \\ ${ }^{2}$ Pediatrics Department, "Gr. T. Popa" University of Medicine and Pharmacy, Iasi, Romania
}

\begin{abstract}
Mycoplasma infection is a common pathology in childhood. The most common involvement known is pulmonary, and the course of the illness is typically mild. Extra-pulmonary involvement include an extremely wide range of manifestations (neurological, cardiac, dermatological, hematological, musculoskeletal, gastrointestinal, renal, uro-genital, ocular), more frequently than we know, with an high incidence, up to $50 \%$. We report two cases of life-threatening extra-pulmoary manifestations caused by Mycoplasma pneumoniae in children. First case is a 17 year old teenager with severe and rapidly progressive form of hemolytic anemia without any anterior respiratory manifestation. Investigations revealed Mycoplasma pneumoniae as etiologic agent. Second case a 3 years old child of with severe sepsis, multi-organ failure, including hematological, in the context of extremely rare co-infection by Mycoplasma pneumoniae and Hantavirus. The onset of illness was with minor respiratory symptoms, but the evolution was rapidly progressive, ultimately leading to the child's death. Both cases have imposed a wide range of investigations for diagnostic and to monitor the treatment. Therapeutic difficulties are presented and discussed.
\end{abstract}

Keywords: Chemolytic anemia, Mycoplasma pneumoniae, child

\section{INTRODUCTION}

Mycoplasma pneumoniae is one of the most frequent etiological agent of community-aquired pneumonia in children, also is implicated in a large number of respiratory infection cases. The real incidence is much higher. The prevalence of Mycoplasma pnemoniae varies among reports, depending on the population studied and the diagnostic methods used (1-2). This has been an estimated as $20-40 \%$ in outpatients and $10-20 \%$ in hospitalized children with community-acquired pneumonia. Controversially, Mycoplasma pneumoniae is the most common agent in children over five years, found in up to $50 \%$ of all pneumonia cases in schoolchildren (3-4).

The most common clinical presentation of $M y$ coplasma pneumoniae infections are mild, and many of them are asymptomatic. A large part of these patients develop extrapulmonary manifestations $(25-50 \%$ - incidence seen in hospitalized patients, including subclinical forms): neurologic (6-
$7 \%)$, cardiac (1-8.5\%), dermatologic (25\%), hematologic $(50 \%)$, musculoskeletal $(14 \%)$, gastrointestinal (25\%), renal and urogenital (rare), ocular (occasional) complications (5-8).

The severity degree of the disease it seems to be correlate with the intensity of the host immune response to infection. Extra-pulmonary complications involving systems and vital organs may be associated with Mycoplasma infection as a result of a direct damage or like an autoimmune response. Extrapulmonary manifestations are sometimes more severe and with a greater clinical significance than the primary respiratory infection (9-12). Hematological manifestations have a significant incidence $\sim 50 \%$, the most common are subclinical. Hemolytic anemia, which also may be associated with Mycoplasma pneumoniae infection is a rare, but extremely severe manifestation. Literature mentions very few cases of this kind (13-19). This may be because of the lack of specific features and a diagnosis that needs serology or PCR. 


\section{CASE REPORT}

We present 2 cases of Mycoplasma pneumoniae infections with extra-pulmonary manifestations in pediatric age, hospitalized in Hemato-Oncology Department of the Emergency Hospital for Children "Sf. Maria", Iasi. Both patients were transferred from the other hospitals, due to the gravity of the cases.

\section{Cas 1}

A 17 years old boy was admitted for sclero-tegumental jaundice, fatigue, asthenia, headache with 4 days onset. He and his family had no past history of any infection in the last 30 days. He denies any drug or tobacco use, toxic exposure. Clinical exam revealed: general state influenced, paleness, sclerotegumental jaundice, normal breath sounds, tachycardia $\mathrm{AV}=120 \mathrm{~b} / \mathrm{min}$, no hepato-splenomegaly, the rest of the examination was unremarkable.

Laboratory tests revealed sever normochromic, normocytic anemia ( $\mathrm{Hb}-5.9 \mathrm{~g} / \mathrm{dl})$, platelets normal, reticulocytosis with peripheral blood smear revealing anisocytosis (microcytes, normocytes, rare macrocytes), unconjugated hyperbilirubinemia (Bi $=5.92 \mathrm{mg} / \mathrm{dl}$ ), inflammatory markers negative, fibrinogen and coagulation tests normal, liver and kidney samples initially normal, indirect Coombs test negative, hipersideremia, increased LDH, low haptoglobin; normal globular resistance, G6PD, $\mathrm{Hb}$ electrophoresis, positive autohemolysis to glucose correction; bone marrow aspiration: effective granulopoiesis; hyperplastic erythropoiesis. Protein electrophoresis, immunogram, total complement, C3 and C4 fraction was performed, within normal limits. Although direct and indirect Coombs repeatedly assessed remained negative, given that hemolysis persisted, and even pronounced (up to total bilirubin $10.28 \mathrm{mg} / \mathrm{dl}$ with indirect bilirubin of $7.0 \mathrm{mg} / \mathrm{dl}$ ), it was considered an immune pathology. Thus, tests that were performed to rule out thyroid impairment, SLE, Wilson's disease - were negative; regarding infectious pathology, viral serology IgM EBV and CMV were negative, $\mathrm{HBsAg}$, hepatitis $\mathrm{C}$ antibody negative, antibodies to human immunodeficiency HIV 1+2 negative. Only antimycoplasma antibody IgM titer was high (titer Ac $=16 \mathrm{U} / \mathrm{ml}$, positive value $>9 \mathrm{U} / \mathrm{ml}$ ). Based on this information, the following diagnosis was considered: Acquired hemolytic anemia, due to subclinical infection with Mycoplasma pneumoniae. Clarithromycin therapy was administrated. Since hemolysis during hospitalization remains active despite corticosteroid therapy associated with re- peated blood transfusions, after 9 days of corticotherapy, it was decided to mount a CVC and initiate plasmapheresis. At the $3^{\text {rd }}$ round of plasmapheresis is was associate immunosuppressive therapy - cyclosporin $200 \mathrm{mg} /$ day orally. The patient gradually improved, but obvious, so the clinical picture (pale skin, disappearance of jaundice sclero-tegumental) and biological (progressive increase in hemoglobin, indirect bilirubin values decrease and marked reticulocytosis). Serum levels of cyclosporine $=42.9$ $\mathrm{ng} / \mathrm{d}$ (effective serum level relative to the dose administered orally for the treatment of immune hemolytic anemia). Discharge (after 30 days of hospitalization in the Department of Hemato-oncology): clinical - good general condition, discreet pale skin, biological parameters normal.

\section{Cas 2}

A 3 years old girl was admitted ICU with general condition extremely serious, toxic infectious syndrome, multiorgan failure (respiratory, renal, hepatic, hematological). The onset 5 days ago, with low grade fever, coryza, cough. The development was to rapidly progressive worsening. Clinical examination at admission: serious overall condition, clouded, paleness, sclero-tegumental jaundice, infiltrate skin, important hematomas on intramuscular puncture places, dyspnea with polypnea, expiratory groan, Sat (-) O2 $=89 \%$, bilateral crepitation, tachycardia $\mathrm{AV}=126 \mathrm{~b} / \mathrm{min}$, abdomen increased volume, moderate hepato-splenomegaly, diuresis preserved. Biological - moderate anemia $\mathrm{Hb}=7 \mathrm{~g} /$ $\mathrm{dl}$, thrombocytopenia $\mathrm{Tr}=45.000 / \mathrm{mmc}$, and neutrophilic leukocytosis, indirect hyperbilirubinemia $\mathrm{Bi}=6.71 \mathrm{mg} / \mathrm{dl}$, urea $=59 \mathrm{mg} / \mathrm{dl}$, inflammatory markers positive, hepatocytolisis, fibrinogen and coagulation tests discrete change. During hospitalization general condition was maintained severe, multi-organ dysfunction included also the heart, patients had 2 episodes of resuscitated cardiac arrest, and an episode of edematous pancreatitis, renal function was subsequently altered, requiring hemofiltration.

Due to the complexity of the case, it required a multidisciplinary team that included: anesthesiologist, pediatric hemato-oncologist, nephrologist, cardiologist, hepatologist, infectionist, pneumologists and pediatric surgeon. For differential diagnosis, multiple investigations have been carried out. They were excluded immune deficiencies, storage diseases, immunological malignancies. Repeated blood cultures were negative. Serologic evaluation regarding infectious pathology: viral serology $\operatorname{IgM}$ EBV and CMV were negative, HBsAg, hepatitis C 
antibody negative, antibodies to human immunodeficiency HIV 1+2 negative, also negative for Borrelia burgdorferi and Parvovirus B19. Anti-mycoplasma antibody IgM titer was high (titer Ac $=16$ $\mathrm{U} / \mathrm{ml}$ positive value $>9 \mathrm{U} / \mathrm{ml}$ ), respectively Ac Hantavirus - IgM titre was positive (titer $1.6 \mathrm{U} / \mathrm{ml}$; positive value $>1.1 \mathrm{U} / \mathrm{ml}$ ). Based on this information, the following diagnosis was considered: Severe sepsis secondary infection with Mycoplasma pneumonia and Hantavirus; multiorgan dysfunction syndrome; cardio-vascular insufficiency; renal failure; hepatic failure; acquired hemolytic anemia; disseminated intravascular coagulation. During the hospitalization, the patient received complex treatment, antibiotico-therapy with broad spectrum. After the Mycoplasma serology was found positive, clarithromycin treatment was associated. She also received multiple blood transfusions, corticosteroids, specific supportive care, immunoglobulins, hemofiltration. For technical reasons, she could not benefit from plasmapheresis. Despite doctors' efforts, progress has been unfavorable, patient dying after a massive intracranial hemorrhage.

\section{DISCUTIONS}

Mycoplasma pneumoniae infection occurs in all age group, being less frequent and more severe under the age of five. Immunity to Mycoplasma pneumonie is transitory and recurrence is frequent. Clinical suspicion of infection with Mycoplasma pneumoniae is essential in severe cases, and healing of patients with lung and extrapulmonary manifestations depends on early diagnosis and specific therapy. Extrapulmonary manifestations could occur before, after, during or in the absence of respiratory symptoms.

Hematological manifestations, other than hemolytic anemia: aplastic anemia, PTI, severe cases of CID, arterial thrombosis, Reynaud's syndrome. True fre-

\section{REFERENCES}

1. Atkinson T.P., Balish M.F., Waites K.B. Epidemiology, clinical manifestations, pathogenesis and laboratory detection of Mycoplasma pneumoniae infections. FEMS Microbiol Rev. 2008 Nov. 32(6):956-73.

2. Michael Joseph Bono, MD, FACEP; Chief Editor: Ryland P Byrd, Jr, MD et all. Mycoplasmal Pneumonia, medscape, 2016

3. Baum S.G. Introduction to mycoplasma and ureaplasma. Mandell GL, Bennett JE, Dolin R, eds. Mandell, Douglas, and Bennett's Principles and Practice of Infectious Diseases. Seventh Ed. Philadelphia, Pa: Elsevier Churchill Livingstone; 2010. 2477-81.

4. Letícia Alves Vervloet, Christophe Marguet and Paulo Augusto Moreira Camargos, Infection by Mycoplasma pneumoniae and Its Importance as an Etiological Agent in Childhood Community-Acquired Pneumonias The Brazilian Journal of Infectious Diseases 2007; 11(5):507-514 quency of hematologic manifestations can reach $50 \%$, but the vast majority are subclinical without significant impact on patient. In conclusion, a wide variety of complications may occur in patients with Mycoplasma pneumonia infection, hemolytic anemia is one of these complications that should be considered in any patient presenting with hemolysis (12-16). Antibiotics are likely to be of limited value in mycoplasma associated hemolytic anemia; however, macrolide treatment has been associated with more rapid resolution of the hemolytic process. Corticosteroids, plasmapheresis and cytotoxic drugs may be tried in refractory cases. Particulars of presented cases: Controversy, the presence of a Coombs test negative in a clear case of autoimmune hemolytic anemia acquired. However, evaluation was performed in both cases after the patients received blood transfusions needed, which could lead to a false negative test. It is also an eloquent illustration of developments of a fulminant hematological manifestation of the infection with Mycoplasma pneumoniae, one in the absence of pulmonary symptoms, the other a case - a coinfection with Hantavirus and with respiratory symptoms.

\section{CONCLUSIONS}

Cases presented highlights the presence of extrapulmonary complications in Mycoplasma pneumonia infection with or without clinical signs of respiratory involvement. This infection could sometimes manifest extremely severe complications, especially at young ages. It might be useful to consider the possibility of a coinfections, especially in cases of severe and refractory to treatment. By drawing attention to extrapulmonary manifestations of Mycoplasma pneumoniae infection, we increase the possibility of a proper diagnosis and appropriate treatment in patients affected.

5. Wiśniewska-Ligier M., Woźniakowska-Gesicka T., Sobańska A., Wierzbicka E. Extrapulmonary complications of Mycoplasma pneumoniae infections, Przegl Lek. 2003; 60(12):832-5.

6. Zafer Kurugol, Serife Sebnem Onen, Guldane Koturoglu, Severe Hemolytic Anemia Associated withMild Pneumonia Caused by Mycoplasma pneumonia, Hindawi Publishing Corporation, Case Reports in Medicine, Volume 2012, Article ID 649850, 3 pg 2

7. Timitilli A., Di Rocco M., Nattero G., Tacchella A., Giacchino R. Unusual manifestations of infections due to Mycoplasma pneumoniae in children. Le Infezioni in Medicina : Rivista Periodica di Eziologia, Epidemiologia, Diagnostica, Clinica e Terapia Delle Patologie Infettive [2004, 12(2):113-117 
8. F.M. Sánchez-Vargas, O.G. Gómez-Duarte, Mycoplasma pneumoniae - an emerging extra-pulmonary pathogen, Clinical microbiology and infection, Volume 14, Issue 2, Feb 2008, Pg 105-115

9. Narita M. Pathogenesis of extrapulmonary manifestations of Mycoplasma pneumoniae infection with special reference to pneumonia. J Infect Chemother. 2010 Jun. 16(3):162-9.

10. Waites K.B., Talkington D.F. Mycoplasma pneumoniae and Its Role as a Human Pathogen. Clin Microbiol Rev 2004; 17:697-728.

11. Domingues D., Nogueira F., Tavira L., Exposto F. Mycoplasmas: What is the role in human infections? Acta Med Port 2005; 18:377-84.

12. Archana Chatterjee, Pediatric Mycoplasma Infections, medscape, 2015

13. J van der Hoek, MD Levin, PHM van der Valk, PM van den Berg, Severe autoimmune haemolytic anaemia in two patients: remember Mycoplasma pneumoniae infection, Neth J. Crit Care, vol.12/4, 2008, pg 167-169

14. Shah S.S. Mycoplasma pneumoniae. Long SS, Pickering LK, Prober CG. Principles and Practice of Pediatric Infectious Diseases. 4th Ed. Philadelphia, PA: Elsevier Churchill Livingstone; 2012. 993-998.
15. Khan F.Y., A Yassin M. Mycoplasma pneumoniae associated with severe autoimmune hemolytic anemia: case report and literature review. Braz J Infect Dis. Feb 2009. 13(1):7

16. D. Kountouras, M. Deutsch, T. Emmanuel, G. Georgiadis J. Koskinas, Fulminant Mycoplasma pneumoniae infection with multi-organ involvement: a case report, Eur. J of Int Medicine, August 2003 Volume 14, Issue 5, Pages 329-331

17. Paramjit Kaur, Sabita Basu, Ravneet Kaur, Gagandeep Kaur, Immune Hemolytic Anemia: A Report of Two Cases, J Lab Physicians. 2009 Jan-Jun; 1(1): 22-24.

18. Wang J.L; Ho M.Y., Shen E.Y. Mycoplasma pneumoniae infection associated with hemolytic anemia--report of one case, Acta Paediatr Taiwan. 2004 Sep-ct; 45(5):293-5

19. F. Daxböck, K. Zedtwitz-Liebenstein, H. Burgmann, W. Graninger, Severe hemolytic anemia and excessive leukocytosis masking mycoplasma pneumonia, Annals of Hematology March 2001, Volume 80, Issue 3, pp 180-182

Conflict of interest: none declared Financial support: none declared 